\title{
Development of small, easy to build and low gas consumming timing RPCs
}

\author{
Miguel Morales* ${ }^{\dagger}$ \\ LabCAF/Univ. de Santiago de Compostela, Spain. \\ E-mail: miguel.moraleseusc.es
}

\section{J.L. Rodríguez-Sánchez}

Department of Particle Phisics/Univ. Santiago de Compostela, Spain.

\author{
N. Teigel \\ Department of Particle Phisics/Univ. Santiago de Compostela, Spain.

\section{Ramos} \\ Department of Particle Phisics/Univ. Santiago de Compostela, Spain.
}

\author{
J. A. Garzón \\ LabCAF/Univ. de Santiago de Compostela, Spain.
}

Timing Resistive Plate Chambers (tRPCs) have become a very popular detectors due to the very good timing capability they offer for covering big surfaces at a very good performance/price ratio. However, such kind of detectors could also be very useful for small experimental setups as an alternative to other methods if their very bulky and uncomfortable gas system could be removed. They are not only very cheap, but they can be built very easily with very simple materials and a few fast electronic components.

Recently, we have opened a research line aiming to develop small and portable sealed tRPCs (sRPCs) and to analyze the evolution of their performances along the time. As a first step, we built a few small one-gap and two-gap detectors and we installed in our laboratory a fast and reliable acquisition setup able to monitorize the main features of the detectors on real time.

We tested some of the developed detectors under different environments and a comparison was made.

XI workshop on Resistive Plate Chambers and Related Detectors - RCP2012,

February 5-10, 2012

INFN Laboratori Nazionali di Frascati Italy

\footnotetext{
*Speaker.

${ }^{\dagger}$ Thanks: Diego Gonzalez (GSI, Germany), Luis Lopes (LIPC, Portugal).
} 


\section{Motivation: Small and portable tRPCs}

Gas ionization detectors are one of the broader family of devices used to detect and to measure the ionization radiation. In some detectors of the family, like the Geiger Muller counters, a sealed volume is filled with the ionizing gas mixture and they are able for working continuously without any visible degradation. Other detectors, like Drift Chambers, have usually a very big size and need very complex gas systems.

RPCs usually run quite well while the gas mixture keeps flowing through the chambers. But relying in a bulky gas system [1] is not always perfect and some effort has been done to decrease this strong dependence, as recycling gas into the system [2], with benefits for the environment and with long run economic savings. Besides, the lack of portability of such bulky systems do not allow thinking about actual portable RPCs.

This work has been focused mainly on the development of small tRPCs able to work without the need of gas flowing systems, as the Geiger Muller counters, but offering very good timing capabilities. Table 1 shows a rough comparison of gas constraints of a typical small Geiger counter and a small sRPC.

$\begin{array}{lll}\text { Typical magnitudes } & \text { sRPC } & \text { Geiger Muller } \\ \text { Example sizes } & 40 \times 40 \times 0.3 \mathrm{~mm}^{3} & 10 \times 40 \mathrm{~mm}^{3} \\ \text { Gas Volume (V) } & \sim 10^{2} \mathrm{~mm}^{3} & \sim 10^{3} \mathrm{~mm}^{3} \\ \text { Edge surface (S) } & \sim 50 \mathrm{~mm}^{2} & \sim 0 \mathrm{~mm}^{2} \\ \text { S/V ratio } & 0.1 \mathrm{~mm}^{-1} & \sim 0 \\ \text { Gas mixture } & \text { R134a/SF6/Isobutane } & \text { Neon/Argon + Halogen gas } \\ \text { Working mode } & \text { Avalanche } & \text { Geiger-Müller } \\ \text { Typical Voltage } & \sim 3000 \mathrm{~V} & \sim 1000 \mathrm{~V} \\ \text { Electric field } & \sim \text { constant } & 1 / \mathrm{r}\end{array}$

Table 1: Geiger Müller counter and sRPC gas chamber comparative.

At this first stage our main goal has been exploring the feasibility of such small and autonomous detectors, starting with the analysis of the evolution of the gas chamber responses. Fur such purpose we build the next prototypes:

1. A one-gap glass RPC, of about $4 \times 4 \mathrm{~cm}^{2}$, with a $0.3 \mathrm{~mm}$ width gas gap

2. Two small two-gaps glass RPCs of $15 \times 5 \mathrm{~cm}^{2}$ : one of them, a standard open RPC, has been operated inside an aluminum box with continuous gas supply and the second one, a sealed RPC, has been operated inside an air tight box filled of gas to avoid any possible degradation by air contamination.

\section{One Gap sRPCs}

Our first step was building a very simple sealed RPC (sRPC) chamber of about $4 \times 4 \mathrm{~cm}^{2}$. One of the first prototypes could be seen in Fig. 1a: just a small one gap gas chamber, two RC circuits, one high frequency analog amplifier and a PT100 temperature sensor were included in the setup. The chamber was glued with an epoxy mixture over a non reactive polymer to avoid the glue to go inside the gap, and to avoid the chamber pollutes by outgassing from these components. 
Later, ten RPCs were handmade by the same method and tested in open gas circuit in order to choose the two better conditioned. Once the electric current felt to a reasonable value of $\sim 0.3$ $\mathrm{nA} / \mathrm{cm}^{2}$, input and output gas tubes of one of the RPCs were cut and glued to ensure the gas chamber stayed sealed. Finally, their operational current (Fig. 1b) and prompt charge distribution (Figs. 1c, 1d), were tested under a ${ }^{22} \mathrm{Na}$ gamma source using the current monitor output of the power supply module and a $1 \mathrm{GHz}$ digital scope respectively. These measurements did not take into account neither pedestal subtraction nor RC overshoot or ionic component, etc.

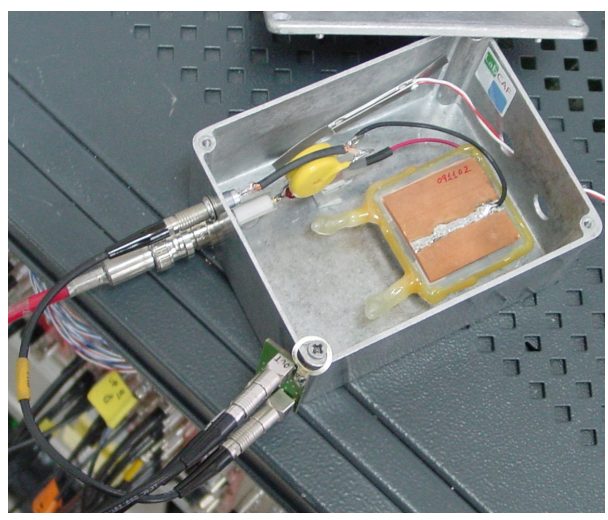

(a) First prototype of a small one gap sRPC.

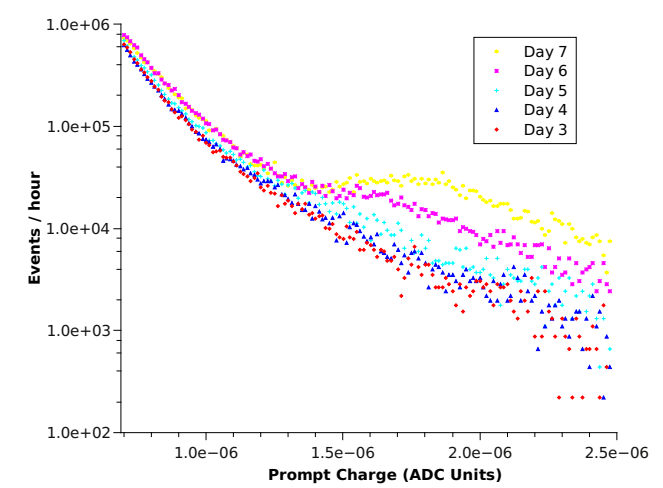

(c) Prompt charge evolutionof a sRPC along first week.

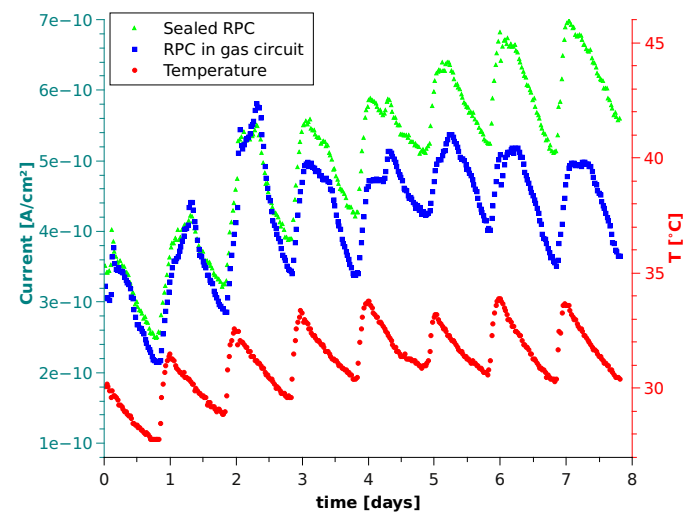

(b) Operational current and temperature evolution of both a sRPC and a reference flowing gas RPC.

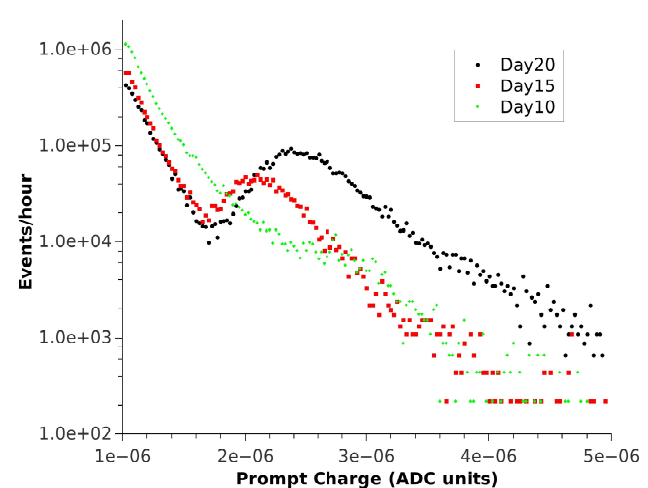

(d) Evolution of the Prompt charge of the sRPC during of three weeks

Figure 1: One-gap sRPC analysis: a) detector setup; b-d) current and prompt charge evolution at different time periods.

Since the third day, the operational current of the sRPC started to grow faster than the one of the reference RPC working in a gas circuit (Fig. 1b). The same effect could be seen at the prompt charge evolution (Figs. 1c, 1d) after a few days working. Gas leaks, internal gas reactions or the outgassing of the materials close to the gap, are the most likely reasons of the observed degradation.

\section{Two Gaps sRPCs}

As a second stage, several two-gap chambers of an approximate size of $5 \times 15 \mathrm{~cm}^{2}$, with four readout channels, were built and operated. In this stage we wanted to open the oportunity of 


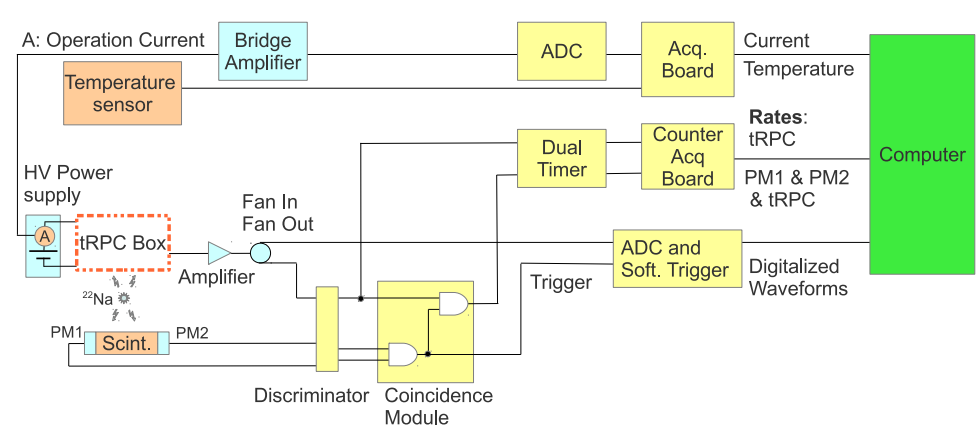

(a) Acquisition setup.

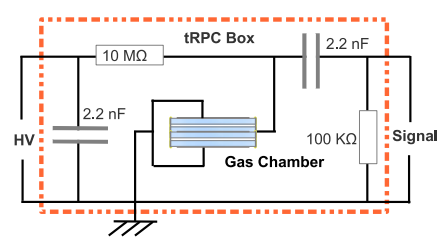

(b) Two-gap RPC configured with two $\mathrm{RC}$ circuits.

Figure 2: Two-gap RPCs acquisition and readout layouts.

analyzing in the future the behavior along greater time periods and having the chance of analyzing also time and position resolution, crosstalk, etc.. For the sake of a better insight over the process, the data acquisition system was significantly upgraded and improved with offline analysis.

\subsection{Acquisition setup and measurements}

The same inbox electronics used with the one-gap sRPCs was used (Fig.[2b]) to deal with coincidences and parallel event counting. Also, to improve the resolution of the operational current monitoring (Fig.[2a]), an electronic add-on was included. It is remarkable that we reached an almost ten times resolution improvement, from $\pm 2 n A$ to $\pm 0.2 n A$, upgrading our acquisition layout with the help of a not very expensive acquisition board and some amplifiers.

It is well known that operational current gives a good insight of the intensity of the gas drifting [3], as long as it is related with the multiplication and attachment coefficient of every specific gas [4]. Therefore, as the gas is the main driven factor of the expected degradation behavior, in order to get some insight of the processes evolving in the gas, a minimum current resolution power is required. Figs. [3a, 3b] show how different gas mixtures give rise to different ionization rates and how different gas degradation evolutions could be discriminated by the new system.

In order to analyze the particular effects arising in a sealed RPC and to be sure that no air contamination appear during our measurements we decided to take the data with two alternative configurations:

1. An open standard two-gap RPC operated inside a normal flowing gas box.

2. A sealed two-gap RPC (sRPC) operated in a tight closed box filled with gas.

\subsection{Discussion}

Fig. 4 shows the evolution of the rate and the operational current of both chambers. The inset shows the corresponding evolution of the mean temperature, that at this stage was not stabilized yet in our laboratory, for a period of one week. We observe how the standard RPC seems to stabilize after the first days of operation keeping it's behavior almost constant despite a small decrease in the mean temperature along the whole period. The sealed RPC seems also to keep their performance 


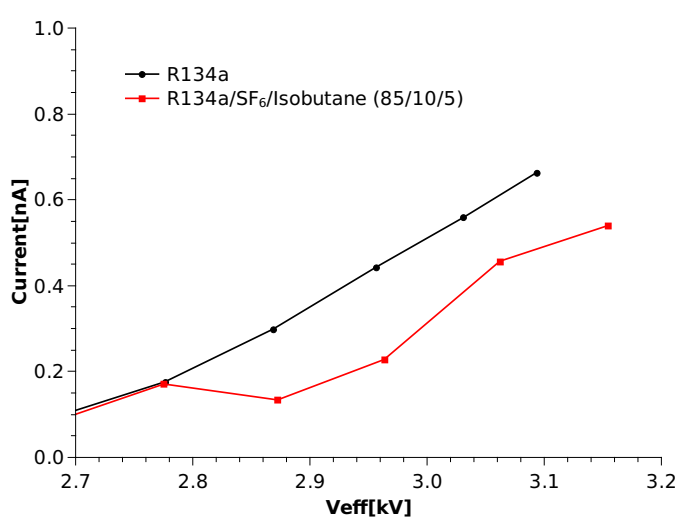

(a) Operational current dependence with the effective electric field for both pure R134a and standard gas mixture.

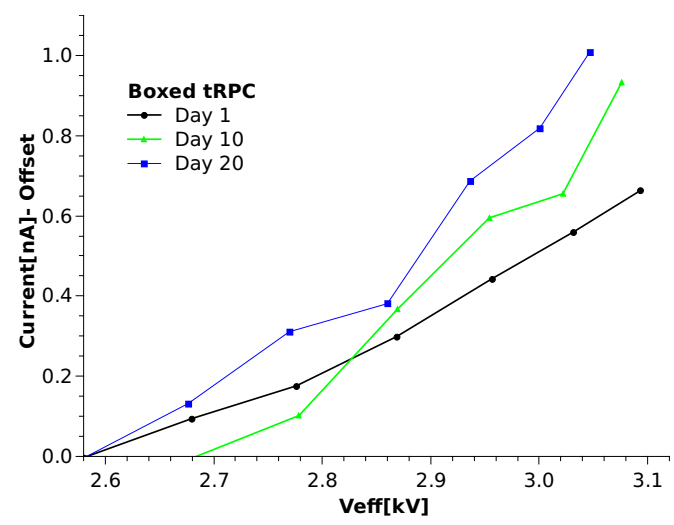

(b) Operational current dependence with the effective electric field for both pure R134a and standard gas mixture.

Figure 3: Analysis of the operational current behavior with both R134a and standard gas mixture with the Boxed RPC.

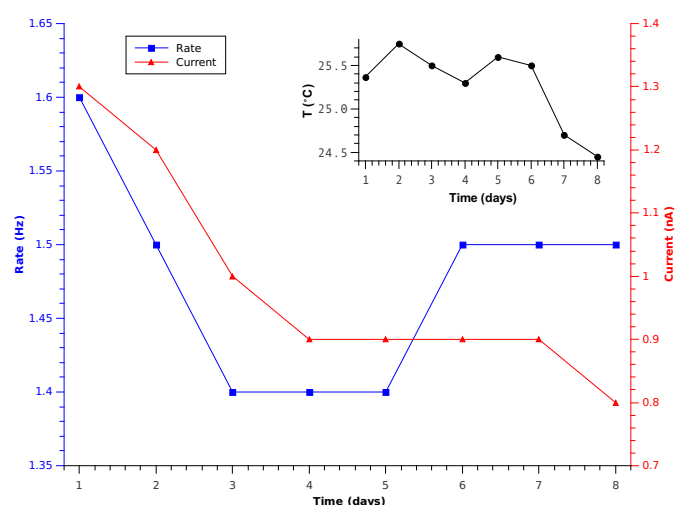

(a) Standard RPC.

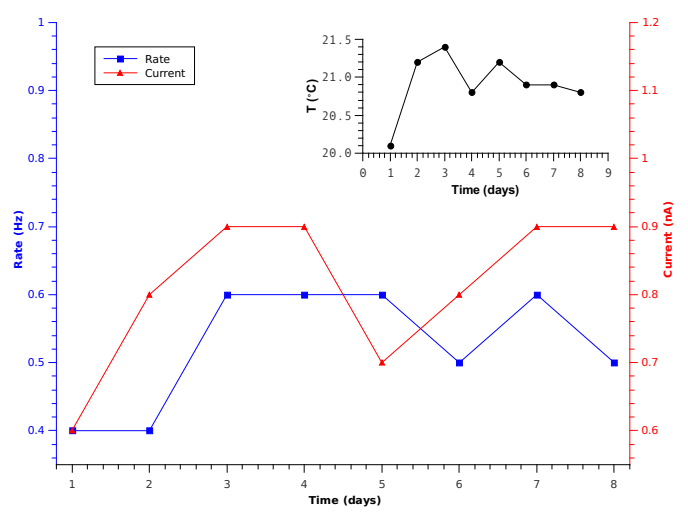

(b) SRPC in a gas box.

Figure 4: Rate and operational current for the different RPCs setups along one week time. The insets show the laboratory temperature as reference.

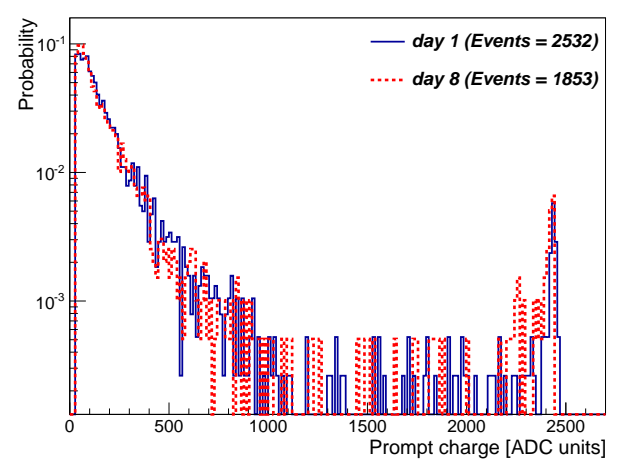

(a) Standard RPC.

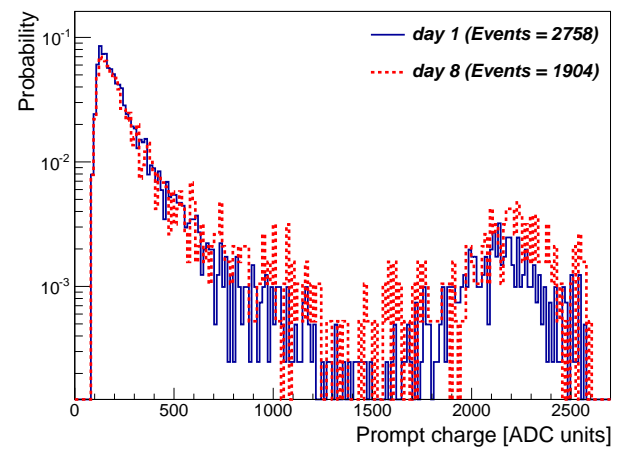

(b) Boxed sRPC.

Figure 5: Evolution of the prompt charge distribution for the different RPC setups. 


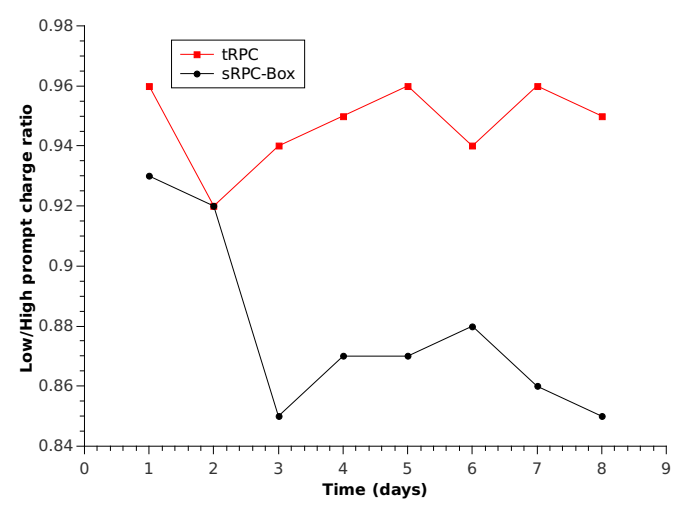

Figure 6: High vs Low prompt charge ratio for boxed sRPC and standard RPC. Threshold between low and high prompt charge is defined around 1300 ADC units.

stable during that time. Fig. 5 shows the evolution of the prompt charges for both detectors. Any of the chambers seem to show any significant evolution of the charge although the sRPC shows since the first day a significant presence of big charge events.

\section{Summary and conclussions}

One-gap and two-gap sealed RPCs (sRPCs) have been built and tested in our laboratory in order to analyze their physical behavior looking at their operational current, rate and prompt charges distributions. At the one-gap prototype, first signs of aging are depicted from the diversion of the operational current from the one provided by a standard RPC. At a second two-gap prototype, their performances were compared with the ones of a reference chamber using an upgraded data acquisition setup and a accurate offline analysis. Preliminary results show that the sealed RPC operated in a box filled with gas, to avoid any air contamination, keeps its performances during at least one week. However, a better temperature stabilization are advisable in order to get more precise conclusions.

\section{References}

[1] S.D. Kalmani, N.K. Mondal, B. Satyanarayana, P. Verma, Avinash Joshi, On-line gas mixing and multi-channel distribution system, NIM A, Volume 602, Issue 3, 1 May 2009, Pages 845-849.

[2] Dominic M. Rossi and Haik Simon, A closed-circuit gas recycling system for RPC detectors, NIM A, 2012, 661, Supplement 1, pp S230 - S233.

[3] S. Ramo, Proc. IRE 27, p.584 (1939).

[4] Werner Riegler, Christian Lippmann, Rob Veenhof, Detector physics and simulation of resistive plate chambers, NIM A, Volume 500, Issues 1-3, 11 March 2003, Pages 144-162. 